\title{
Efficient Approach for Analyzing the Driver's Vigilance
}

\author{
Belkacem Abbadi \\ MSE Laboratory \\ University of B. B. Arreridj \\ BP 64 - 34030 - Algeria
}

\author{
Djamel Boubetra \\ MSE Laboratory \\ University of B. B. Arreridj \\ BP 64 - 34030 - Algeria
}

\author{
Messaoud Mostefai \\ MSE Laboratory \\ University of B. B. Arreridj \\ BP 64 - 34030 - Algeria
}

\begin{abstract}
Road accidents, due to the tiredness or the distraction of the drivers, unfortunately became more serious than a war. Although more or less effective solutions were developed to solve this problem, these last remain in general constraining and/or sensitive to the variations of lighting. This paper presents a new approach for analyzing the driver's vigilance based on motion analysis of an on-head reflecting point. Proposed solution is non-intrusive and easily adaptable to all types of vehicles. Moreover, developed tracking algorithm has a low computational complexity and is therefore well suited for a hardware implementation to suit real time driving constraints.
\end{abstract}

\section{General Terms}

Motion analysis, video processing, computer vision, real-time, vehicular technology, video tracking.

\section{Keywords}

Vehicles safety, vigilance analysis, drowsiness, distraction, head motion.

\section{INTRODUCTION}

Road accidents, due to the fatigue or the hypovigilance of the drivers, do not cease growing, causing unfortunately more and more deaths and handicapped people. Although important human and material means were mobilized by the governments in order to limit this plague (become as harmful as a war), the accidents do not cease increasing with the increase of the vehicles power and number. Besides, a considerable technological effort is made by the manufacturers and research laboratories in order to find practical solutions. These lasts would offer more safety to the drivers by warning them at the right time of the risk that surrounds them and this is according to the situations they are in [1]. Although they are powerful, the suggested solutions are not quite practical in terms of meeting some constraints other than the performance, that is, universality and intrusiveness. Since the main goal behind these efforts is to make driver assistance systems accessible for all vehicle's owners, it is important that these systems must be as simple as possible. Thereby, it can be automatically fitted to all existing vehicles and do not remain an option of the new high class vehicles. On the other hand, the delicate driving circumstance requires that the driver should not be hampered by the system, especially when the vehicle is moving in a high speed [2]. On the basis of the principle "simplicity is the summit of complexity", an effective and non-constraining solution for analyzing the driver's vigilance is proposed, in this work. In addition to its robustness against light variations, the proposed method is non-intrusive and easily adaptable to all types of vehicles.

\section{STATE OF THE ART}

Over the last years, vehicular safety has gained a significant benefit from the dramatic development in vehicle automation [3]. Among the recent achievements that aim to enhance driver's safety, systems of monitoring drivers' level of vigilance have attracted a privileged attention of researchers and vehicle companies. In contrast to other automated systems, that may exist inside vehicles, the challenging drawback of designing efficient vigilance monitoring systems is to find out fast and effective methods to measure and analyze the state of conductors at real driving conditions [2]. To this end, many systems have been developed. Most of them are based on vehicle-based measures, behavioral or physiological measures.

Although they guarantee satisfactory simulation results, vehicle based-measurement methods (such as the steering wheel movement or standard deviation of lane position) depend directly on road marking and lighting conditions [4]. Methods based on physiological measures (ECG, EEG, oxygen saturation in the blood, etc.) are very reliable and allow robust and precise driver's state analysis. However, the latter are very constraining and not suitable for a general public use [5]. Besides being intrusive, they need sophisticated acquisition and complex processing [6]. Despite the wide employment of the methods based on behavioral measures, they provide less performance compared to that based on physiological attributes. They are non-intrusive and often make use of computer vision approach to analyze specific cues that may indicate drowsiness state [7]. These lasts are mainly detected on the driver's face using a simple camera. As a key benefit, computer vision approach is nonintrusive since it allows a remote measurement. Moreover, it is possible to simultaneously acquire many parameters through one sensor, which results in a cost-effective characterization of the driver's state. Various works around this approach are reported in the literature [8]. The eyes blinking and yawning frequency are two of the most useful parameters that relate to the driver's level of vigilance [7]. For this purpose, different attempts are presented in [9][10][11]. These lasts aim efficiently -and in real time- to locate eyes and mouth, and track their movements in video stream. Decisions about drowsy state are made according to the yawning frequency and/or the PERCLOS value (rate of eyes closure within a given time). However, these approaches are very sensitive to variations of lighting conditions and face poses [12]. Aiming to work out this problem, Vitabile et al. [6] have presented a drowsiness detection system based on Infrared lighting. After a relevant segmentation, eyes are detected trough clipping and morphological operations. PERCLOS is employed to infer driver's level of fatigue. Once again, worst results may be obtained in case of conductors wearing glasses. Hypovigilance may be detected through the driver's head pose. Since head movement may be simply tracked, even in weak lighting, Vural et al. [13] have proposed a system based on eyes blinking, yawning 
frequency, and head motion. This last is acquired using an accelerometer. Machine learning has been used to make decisions. The system presented in [12] exploits both eyelid closure and head pose. Viola and Jones algorithm has been employed to locate and track the face. SVM technique has been used to measure eyes closure, and Neural Networks are involved to detect the driver's state. Works presented in [14] and [15] are based on just 3D head orientation. In [14], authors have established rules to automatically determine the head orientation by exploiting three face characteristic points. Interest points are located by using Haar features method and Harris detector. Thus, drowsiness is captured through analyzing the head movement. In [15], authors have developed a new technique to track the head during driving. This is done by collecting head positions by four infrared sensors which are mounted on the headrest of the driver's seat.

\section{BASIC PRINCIPLE}

In addition to the mechanical problems, the temporary hypovigilance of the driver on the road is the main cause leading to the car control loss. This dangerous situation can be due to a beginning of drowsiness or the distraction of the driver.

The driver's state can be evaluated by a real time analysis of the position of the head and/or the state of the eyes. The behavioral methods are well suited for such analysis. Unfortunately, they present limits in the case of variable lighting, or if the person wears glasses. To overcome these problems, we propose in this work, to provide the drivers with glasses equipped with a reflecting point, easy to follow and analyze throughout driving time with a simple camera.

As essential as the safety belt, wearing glasses with a reflecting point is not constraining for the majority of the drivers. For people who do not carry glasses, neutral glasses or an on-head equipped with a reflecting point can be used (see Figure1).

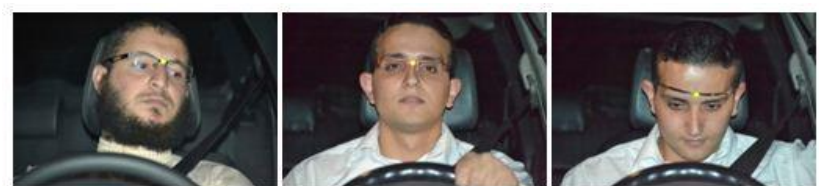

Fig 1: Reflecting point dispositions

As presented in Figure 2, a digital video camera is placed in front of the conductor. The disposition of the last will depend on the car and the conductor's characteristics. The goal is to have a clear view with the conductor head nearly at the center of the filmed scene. The reflecting point video processing can start automatically (according to preset conditions: state of the vehicle, speed, day/night, etc.), or activated manually by the conductor himself.
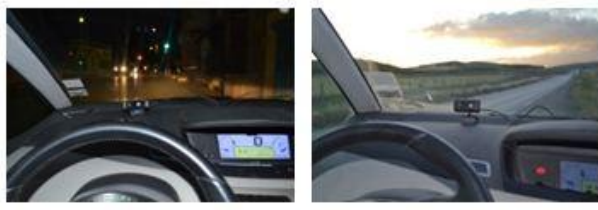

Fig 2: Examples of camera placements

In what follows, we will show that a dynamic motion analysis of this reflecting point, allows having precious information regarding the driver's head position and consequently the degree of his vigilance.

\section{PRELIMINARY EXPERIMENTAL TESTS}

The primary prototype is composed of a simple webcam linked to a laptop PC. Recorded videos are actually treated off-line with a developed MATLAB program. The last allows the extraction of successive reflecting point positions and the computation of the corresponding velocities $\left(x(t), y(t), V_{x}(t)\right.$ and $\left.V_{y}(t)\right)$.

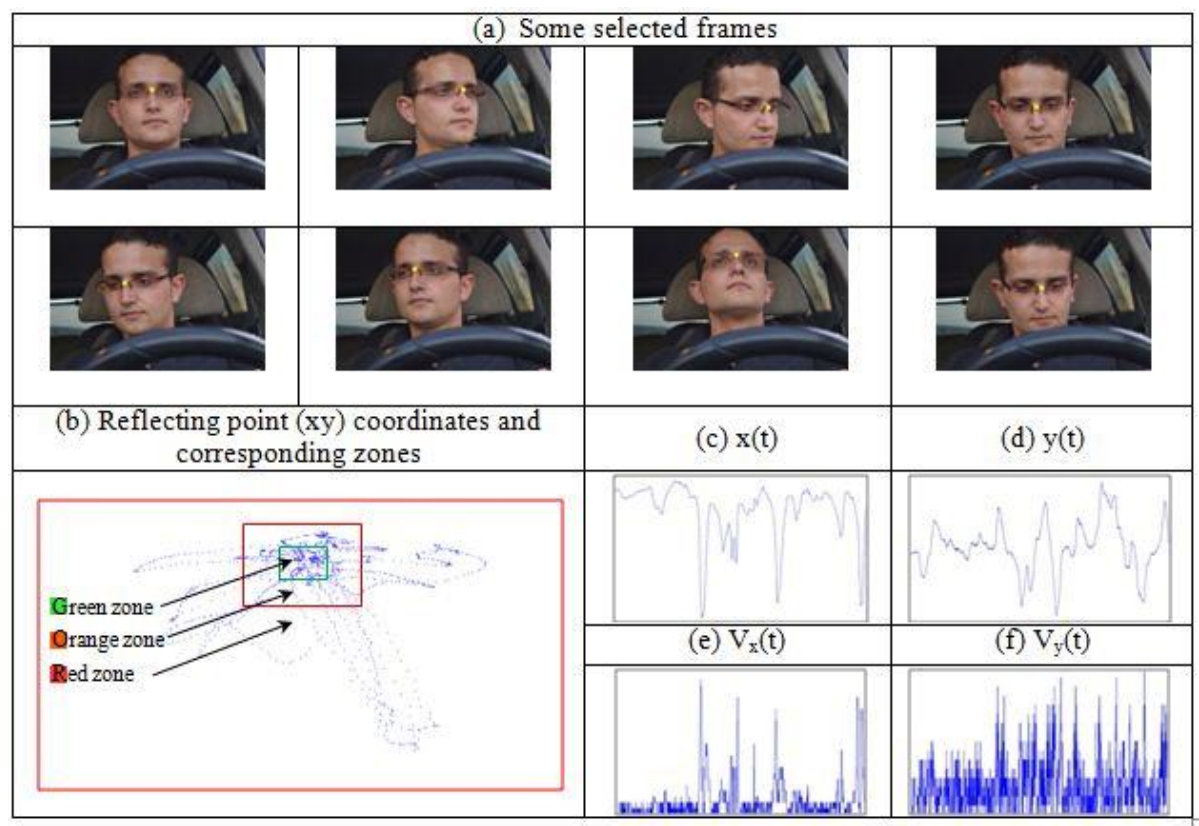

Fig 3: Example of acquired frames and their corresponding velocities

First, each video frame is segmented into two regions, by using the color of the reflecting point as a threshold. Then, the centroid coordinates $(x, y)$ of the region corresponds to the reflecting point is retrieved using "regionprops" dedicated
MATLAB function. In the end of this process, one obtains a $2 \times T$ table; with $T$ is the number of frames of a recorded video sequence. 
In order to visualize the point movement which reproduces the driver's head movement, the $T$ coordinates represented by the obtained table are mapped in a $2 D$ plan. To allow more precise analysis, the evolution of abscises and ordinates and their related velocities along $t$ dimension are needed $(x(t), y(t)$, $V_{x}(t)$ and $\left.V_{y}(t)\right)$. Velocities are obtained through a simple subtraction of each two successive $x$ or $y$ coordinates.

An example of obtained results is presented in Figure 3. From the acquired reflecting point coordinates (Figure 3.b), three possible zones can be defined:

- The green zone " $G$ " for centered positions of the head,

- The orange zone "O" for slightly off-centered positions of the head,

- The red zone " $\mathrm{R}$ " for highly off-centered positions of the head.

Thus, in case the red zone is detected, a dedicated signal alarm (luminous or sound) will be sent to the driver to alert him.

It is important to note that drowsiness detection requires a precise treatment and analysis of the velocities signals in order to evaluate the speed of the tracked reflecting point which in this dangerous case varies brutally [16][17].

Figure 4 presents a simulated drowsiness case and its corresponding velocity curves. If we precisely focus on acquired curves, we can detect the zone where only $y(t)$ and $V_{y}(t)$ curves varies brutally. This zone precisely corresponds to a drowsiness state.
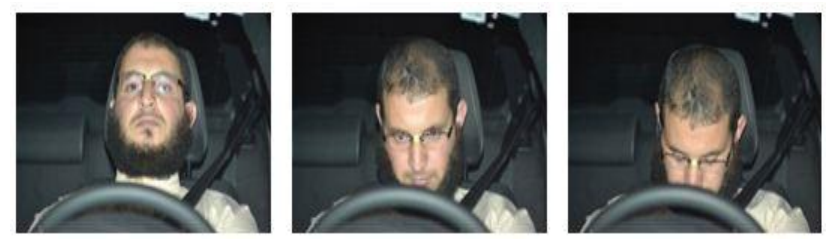

(a) Selected drowsiness frames

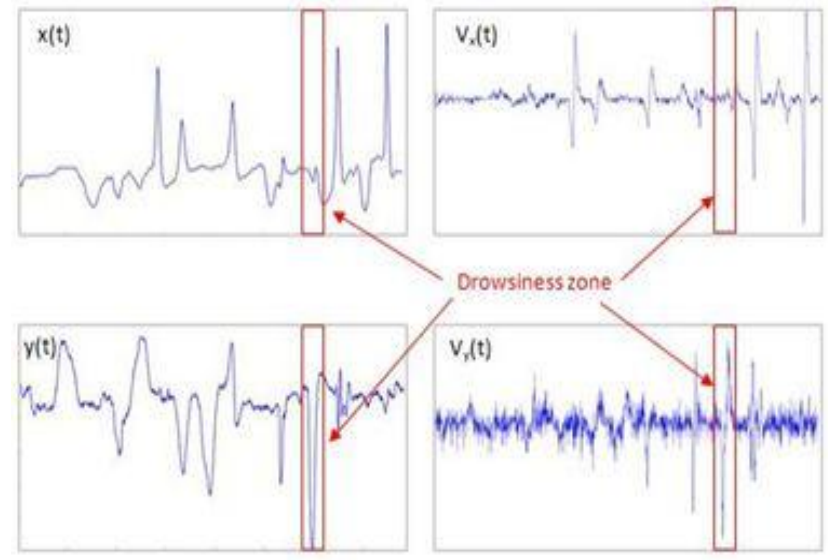

(b) Corresponding curves

Fig 4: Simulated Drowsiness case

Actually, the spherical form of the chosen reflecting point does not allow the recognition of the orientation of head. A reflecting point with several facets can be used to allow a precise analysis of the position and the orientation of the head.

\section{CONCLUSION}

A non-constraining driver's vigilance analyzing method is presented. The last consists of providing the drivers with glasses or an on-head equipped with a reflecting point, easy to follow and analyze with a video camera.

Besides being non-intrusive and robust against light changes, the proposed solution is easily adaptable to all types of vehicles. In terms of computing overhead, the process of retrieving the driver's head movement involves simple image processing operations that can simply respect real-time deadlines of the system. Preliminary experimental tests have shown the effectiveness of the proposed method in the driver's state characterization.

However, more work remains necessary to ensure a precise and intelligent analysis of retrieved data, allowing thus efficient and fast drowsiness detection. To suit real time driving constraints, a hardware module will be developed to allow an online head position computation.

\section{REFERENCES}

[1] Sahayadhas, A., Sundaraj, K., and Murugappan, M. 2012. Detecting Driver Drowsiness Based on Sensors: A Review. Sensors. 12 (Dec. 2012), 16937-16953.

[2] Kleinberger. T., Jedlitschka, A., Storf, H., SteinbachNordmann, S., and Prueckner, S. 2009. An approach to and evaluations of assisted living systems using ambient intelligence for emergency monitoring and prevention. In Proceedings of the 5th International Conference, UAHCI, Part II.

[3] Rakotonirainy, A. and Tay, R. 2004. In-vehicle ambient intelligent transport systems (i-vaits): Towards an integrated research. In Proceedings of the 7th international IEEE conference on intelligent transportation systems.

[4] Liu, C.C., Hosking, S.G., and Lenné, M.G. 2009. Predicting driver drowsiness using vehicle measures: Recent insights and future challenges. Journal of safety research. 40 (Aug. 2009), 239-245.

[5] Akin, M., Kurt, M., Sezgin, N., and Bayram, M. 2008. Estimating vigilance level by using EEG and EMG signals. Neural Computing and Applications. 17 (June 2008), 227-236.

[6] Vitabile, S., De Paola, A., and Sorbello, F. 2011. A realtime non-intrusive FPGA-based drowsiness detection system. Journal of Ambient Intelligence and Humanized Computing. 2(Dec. 2011), 251-262.

[7] Jixu Chen, J. and Ji, Q. 2012. Drowsy Driver Posture, Facial, and Eye Monitoring Methods. In Handbook of Intelligent Vehicles, Eskandarian, A.

[8] Smith, P., Shah, M., and Vitoria, L.N. 2003. Determining driver visual attention with one camera. IEEE Trans. Intell. Transport. Syst. 4 (Dec. 2003), 205-218.

[9] Hemadri, V. B. and Umakant, P. K. 2013. Detection of Drowsiness Using Fusion of Yawning and Eyelid Movements. In Proceedings of the Third International Conference, ICAC3.

[10] Alioua, N., Amine, A., Rziza, M., and Aboutajdine, D. 2011. Driver's Fatigue and Drowsiness Detection to Reduce Traffic Accidents on Road. In Proceedings of the 14th International Conference, CAIP, Part II. 
[11] Choong Lai, K., Wong, M. L. D., and Islam, S. Z.2013. A HW/SW Co-Design Implementation of Viola-Jones Algorithm for Driver Drowsiness Detection. In Future Information Communication Technology and Applications. Lecture Notes in Electrical Engineering Volume 235, Jung, H. K., Kim, J. T., Sahama, T., Yang, C. H.

[12] Flores, M. J., Armingol, J. M., and De La Escalera, A 2010. Real-Time Warning System for Driver Drowsiness Detection Using Visual Information. Journal of Intelligent \& Robotic Systems. 59(Aug. 2010), 103-125.

[13] Vural, E., Cetin, M., Ercil, A. L., Gwen, Bartlett, M., and Movellan, J. 2007. Drowsy Driver Detection Through Facial Movement Analysis. In Proceedings IEEE International Workshop, HCI.

[14] Akrout, B. and Mahdi, W. 2013. Vision Based Approach for Driver Drowsiness Detection Based on 3D Head
Orientation. In Multimedia and Ubiquitous Engineering, MUE 2013, Park, J. J., Kee-Yin Ng, J., Jeong, H.Y., Waluyo, B.

[15] Lee, D., Oh, S., Heo, S., and Hahn, M. 2008. Drowsy Driving Detection Based on the Driver's Head Movement using Infrared Sensors. In 2nd International Symposium on Universal Communication (ISUC '08).

[16] Barr, L., Popkin, S., and Howarth, H. 2009. An Evaluation of Emerging Driver Fatigue Detection Measures and Technologies. Technical Report. U.S Department of Transportation.

[17] J, Q., Lan, P. and Zhu, Z. 2004. Real-time non-intrusive monitoring and prediction of driver fatigue. IEEE Transactions on Vehicular Technology. 53(July 2004), $1052-1068$ 